\title{
Posterior Reversible Encephalopathy Syndrome in Primigravida with Severe Pre-eclampsia and Hellp
}

\author{
Sevim Özden ${ }^{1 *}$, Christina Lindegaard Poulsen ${ }^{2}$ and Mohammed R \\ Khalil $^{2}$ \\ ${ }^{1}$ Department of Anesthesiology, Sygehus Lillebaelt, Kolding, Denmark \\ ${ }^{2}$ Department of Obstetrics and Gynaecology, Sygehus Lillebaelt, Kolding, Denmark \\ *Corresponding Author: Sevim Özden, Department of Anesthesiology, Sygehus \\ Lillebaelt, Kolding, Denmark.
}

Received: October 22, 2020

Published: Dcember 08, 2020

(C) All rights are reserved by Sevim Özden., et al.

\begin{abstract}
Posterior reversible encephalopathy syndrome (PRES) is a neuroradiologic condition characterized by a range of symptoms such as headache, nausea, vomiting, blurred vision, altered consciousness, seizures, and focal neurological symptoms. It can be associated with HELLP syndrome. In this case report, we present 29 years old primigravida at 36+6 weeks' gestation with HELLP syndrome and severe preeclampsia with loss of vision on both eyes. The patient was further examined with a CT brain scan, which initially indicated that she had posterior reversible encephalopathy syndrome (PRES). Neuro radiologist provided a second interpretation of the CT brain scan with no sign of PRES. However, the clinical presentation indicated signs of PRES and the patient got treatment with antihypertensive and Magnesium Sulphate at the intensive care unit and had uneventful caesarean section performed. This case report emphasizes the importance of early recognition, correct treatment, and management to prevent further complications.
\end{abstract}

Keywords: Hypertension; Pre-eklampsia; HELLP; Pregnancy; PRES; Loss of Vision

\section{Introduction}

Preeclampsia is a severe and potential life-threatening complication in pregnancy. It is defined by gestational hypertension accompanied by proteinuria and/or signs of organ dysfunction. The condition is classified into mild, moderate, or severe preeclampsia. The latter could possibly cause either generalized convulsions also known as eclampsia or HELLP syndrome characterized by hemolysis, elevated liver-enzymes, and low platelets. HELLP syndrome is a biochemical diagnosis that appears either without any symptoms or as part of severe preeclampsia and should, therefore, be considered an independent variation of severe preeclampsia. The treatment of preeclampsia is antihypertensive medication and magnesium sulfate at signs of eclampsia or HELLP. The ultimate treatment of severe preeclampsia is delivery of the baby; however, stabilization of the mother is crucial before intervention [1].

Posterior reversible encephalopathy syndrome (PRES) is a neuroradiologic condition characterized by among other symptoms such as headache, nausea, vomiting, blurred vision, altered consciousness, seizures, and focal neurological symptoms [2,3]. HELLP syndrome can contribute to the development of PRES syndrome that is an underdiagnosed complication [4]. The pathophysiology of PRES still remains unclear and is poorly defined although theories have been suggested [5]. One of these is the loss of cerebral autoregulation due to abrupt high blood pressure changes with endothelial damage due to the excessive pressure and breakdown of the blood-brain barrier. The result is cerebral hyper perfusion with protein and fluid extravasation causing vasogenic edema [6].

PRES can be seen in the setting of drug toxicity, sepsis, SLE, renal failure, immunosuppressive therapy and autoimmune diseases. Differential diagnoses are intracerebral hemorrhage, CNS-infection, epilepsy, sinus thrombosis, posterior circulation infarct, and ischemic apoplexy. In some cases, patients do not have hypertension, and the postulate is that, it is a rapid change in blood pressure and not the absolute blood pressure that is important for development of the condition [7]. 


\section{Case Presentation}

A 29 years old primigravida at $36+6$ weeks' gestation was admitted to Obstetric department, Kolding Hospital, Denmark, with HELLP syndrome. The patient failed to attend two previous followup appointments with the obstetrician and midwife. Due to general vulnerability and the unborn child diagnosed at prenatal routine ultrasonographic follow-up with lip cleft palate, the patient had follow-up at specialized outpatient clinic. At admittance, she had severe preeclampsia, and the blood pressure was measured multiple times and elevated up to systolic 220. Her main complaints were nausea, vomiting, headache, and epigastric pain. Standard panel of preeclampsia blood tests, which included full blood count, liver function test, urea, electrolytes, and coagulation parametres, were obtained. Analyses of urine showed proteinuria 3+. Blood tests (normal levels in parentheses) shoved creatinine level of 128 (45-90), alanine transaminase of 965 (10-45), bilirubin of 116 (525), lactate dehydrogenase of 1400 (105-205), hemoglobin level of 6.4 (7.3-9.5), platelet count of 31 (168-400), antitrombin of 0.75 (0.80-1.20), and D-dimer of $>20(<0.5)$. During the hospitalization, she suddenly developed loss of vision on both eyes, and she was transferred to the Intensive Care Unit and treated with Magnesium Sulphate infusion. Blood pressure was closely monitored and treated with intravenous Labetalol and Nifedipine. CT of the brain showed signs of PRES syndrome and no signs of bleeding, infarct nor thrombosis. The signs of PRES were hypodense changes in white matter substance bilaterally, especially in occipital area, and general edema. Second interpretation of the CT-images was performed by a neuro radiologist which didn't show any sign of PRES. She was managed preoperatively under universal anesthesia and had an uneventful caesarean section performed with a healthy baby born.

\section{Outcome and fellow-up}

The loss of vision was completely resolved after normalizing of the blood pressure. The patient was discharged from the hospital with near-normally blood tests. A follow-up consultation was managed weeks after according to procedures of complicated pregnancies/deliveries.

\section{Discussion and Learning Points}

Symptoms and MR of the brain set diagnosis of PRES. Only MR can distinct PRES from differential diagnosis [8]. In a prospective study enrolled with 104 women with eclampsia showed that parietal and occipital lobes were most frequently affected, however, any part of the brain can be affected [9]. In the present case, the patient had a CT scan performed of the brain. The radiologists suggested PRES without a definite diagnosis concluded. Because CT scan of the brain in this case showed no signs of bleeding, infarct nor thrombosis, PRES was the most obvious diagnosis and the patient was treated accordingly. Other similar cases have shown delay in recognition and adequate treatment of PRES because of initial CT scan and secondary MR scan [10]. Early recognition and treatment is crucial to avoid a permanent brain damage. Aaen et al. describes in a case report the importance of early recognition of PRES [8]. The patients can have a broad range of symptoms and presentations, which makes the diagnose difficult. Delayed diagnosis may lead to irreversibility and even mortality and morbidity. If a patient presents with earlier mentioned symptoms and loss of vision during preeclampsia PRES must come into mind. The treatment is mainly supportive. Patients will improve by lowering blood pressure; however, in order to avoid cerebral ischemia, the blood pressure reduction should not exceed $25 \%$ of the presenting value within few hours. Antihypertensive agents such as Labetalol and Nifedipine can be used safely following national guidelines. Delivery of the baby and Magnesium Sulphate infusion is sufficient treatment in most cases with preeclampsia [7].

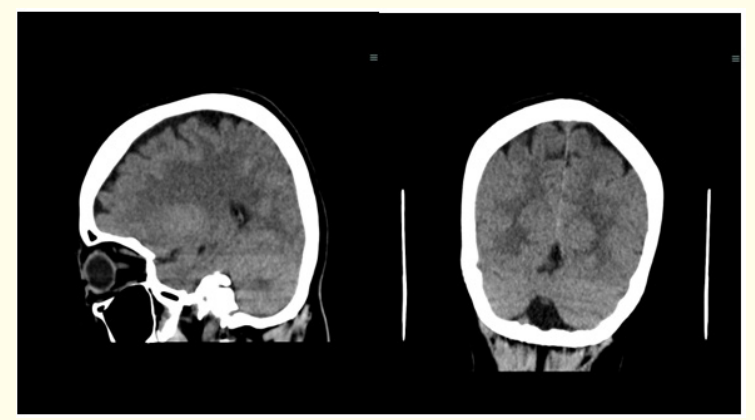

Figure 1: Normal CT-brain scan of the patient.

\section{Conclusion}

Early recognition and treatment of Posterior reversible encephalopathy syndrome(PRES) is crucial to avoid a permanent brain damage. Delayed diagnosis may lead to mortality and morbidity and early treatment is crucial for recovery and reversibility. Symptoms and MR of the brain set diagnosis of PRES and the treatment is mainly supportive. Antihypertensive drugs, delivery of the baby and Magnesium Sulphate infusion is sufficient treatment in most cases with preeclampsia. 


\section{Conflict of Interest}

We have no conflicts of interest to disclose.

\section{Bibliography}

1. Lise Hald Nielsen., et al. "Hypertension and preeclampsia". DSOG guideline (2018).

2. Seda Ates., et al. "Posterior reversible encephalopathy associated with HELLP syndrome: A case report and review of the literature". Neurology Asia 20.1 (2015): 91-94.

3. Screenivasa Rao Sudulagunta., et al. "Posterior reversible encephalopathy syndrome (PRES)". Oxford Medical Case Reports 4 (2017).

4. Marlene Fischer and Erich Schmutzhard. "Posterior reversible encephalopathy syndrome”. Journal of Neurology 264 (2017): 1608-1616.

5. Moulay Abdellah Babahabib., et al. "Posterior reversible encephalopathy syndrome in patient of severe preeclampsia with Hellp syndrome immediate postpartum". Pain African Medical Journal (2015): 21-60.

6. Jeniffer E Fugate and Alejandro A Rabinstein. "Posterior reversible encephalopathy syndrome: clinical and radiological manifestations, pathophysiology and outstanding questions". The Lancet Neurology 14.9 (2015): 914-925.

7. Terry A Neill. "Reversible posterior leukoencephalopathy syndrome”. UpToDate. Wolters Kluwer.

8. Anne Albers Aeen. Jørgen Jeppesen. Hayder Obaid. Hans henrik Bülow. Post partum posterior reversibelt encefalopati-syndrom. Ugeskr Læger (2015): 177.

9. Anand Kumar Verma., et al. "Posterior encephalopathy syndrome in women with eclampsia: Predictors and outcome". Elsevier 10 (2017): 74-82.

10. Ilda Amirian., et al. "Posterior reversibel encefalopatisk syndrom hos en gravid”. Ugeskr Læger 180 (2018): V01180049

\section{Assets from publication with us}

- Prompt Acknowledgement after receiving the article

- Thorough Double blinded peer review

- Rapid Publication

- Issue of Publication Certificate

- High visibility of your Published work

Website: www.actascientific.com/

Submit Article: www.actascientific.com/submission.php

Email us: editor@actascientific.com

Contact us: +919182824667 